Reviewer's Abstract for 44th AIAA Aerospace Sciences Meeting and Exhibit, 9-12 January 2006, Reno, Nevada

\title{
AIRBORNE TURBULENCE DETECTION SYSTEM CERTIFICATION TOOL SET
}

D.W. Hamilton and F.H. Proctor NASA Langley Research Center Hampton, VA 23681-2199 USA d.w.hamilton@1arc.nasa.gov /Fax+757-864-8858

Unexpected in-flight encounters with turbulence result in frequent injuries and infrequent fatalities. To alleviate this problem and contribute to the mission of NASA's Aviation Safety Program, technologies are being investigated to detect and warn of hazardous in-flight turbulence. Under the sponsorship of the Turbulence Prediction and Warning Systems (TPAWS) element of NASA's Aviation Safety program, a radar-based airborne turbulence-detection system (ATDS) has been developed and tested, which improves upon existing commercially available systems. The new system utilizes algorithms and hazard metrics developed for use with existing airborne windshear radar, and are expected to warn of hazardous turbulence even in regions with weak radar reflectivity (i.e. 5-15 dBZ). Industry is expected to market similarly improved ATDS components in the near future. Since the new systems may incorporate alerting functionality, requiring flight crews to take procedural action in the event of a potentially hazardous turbulence encounter, industry is expected to file for FAA certification. To prepare for this activity, NASA is working with the FAA to develop a certification methodology and an accompanying set of tools to evaluate the performance of industry built ATDS.

As a part of the proposed certification methodology, NASA has developed an end-to-end simulation "tool set" designed to evaluate the performance of a candidate 
ATDS. The evaluation would be based on the ability of the candidate ATDS to meet performance standards set by the FAA. To evaluate its performance, the candidate ATDS must make a large number of turbulence predictions under a variety of atmospheric and aircraft conditions. Testing of such a system solely based on actual flight experiments is costly and difficult to evaluate due to the typically unknown characteristics of the atmosphere and aircraft state. Therefore, the "tool set", consisting of model-generated atmospheric turbulence data sets, hazard estimation models, aircraft performance models, a radar simulation software, and an automated scoring routine, will provide the controlled environment necessary for such an evaluation.

In the "tool set", the atmospheric data sets represent convectively induced turbulence environments where aircraft encountered hazardous turbulence. This paper will describe the development of three data sets and their validated with observed data. The paper will also demonstrate the "tool set" with examples of flights through the said data sets. In the examples, turbulence hazard predictions will be made using the following elements of the "tool set"; the Airborne Doppler Weather Radar Simulation $(A D W R S)^{1}$, the NCAR Efficient Spectral Processing Algorithm (NESPA) ${ }^{2}$, the Moving Box Method, ${ }^{3}$ and Bowles' flight dynamics model ${ }^{4}$. Comparisons of the predictions will be made for a variety of aircraft types, weights, airspeeds, and altitudes. Radar based hazard predictions will be scored based on a simple hazard metric, or 'truth' measurement, which is derived from the aforementioned Moving Box Method.

In all examples, the atmospheric data sets are comprised of gridded threedimensional velocity fields and radar reflectivity of thunderstorm environments. These environments have been modeled using the Terminal Area Simulation System (TASS) ${ }^{5}$. 
Two of the events are associated with turbulence encounters during two separate NASA Turbulence Flight Experiments and the third with an event on an aircraft participating in the Flight Operations Quality Assurance Program (FOQA).

An example of how turbulence hazard predictions are made is presented here. The event chosen is a turbulence encounter that occurred during one of NASA's flight experiments; Event 232-10. The turbulence measurement, or turbulence level, is quantified in terms of the root mean square of the normal load acceleration over a 5 second window, $\sigma_{\Delta \mathrm{n}}$ (units in $g$ 's). Measurements of $\sigma_{\Delta \mathrm{n}}$ exceeding 0.3 , constitutes severe turbulence and is equivalent to peak normal accelerations, $\Delta \mathrm{n}$, exceeding one $g .{ }^{6}$ Figure 1 shows the airborne radar reflectivity and predicted turbulence hazard, $\sigma_{\Delta \mathrm{n}}$, from the actual event. ADWRS simulated radar reflectivity and NESPA predicted hazard compare well with those acquired from the actual flight, as shown in Figure 2. The peak hazard magnitude and location predicted by the radar simulation (right panel in Figure 2) compare well with the 'truth' depicted in Figure 3. A flight through the data set using Bowles' flight dynamics model is shown in Figure 4. In the plot, the strong plume is evident with a peak vertical velocity of nearly $12 \mathrm{~m} / \mathrm{s}$. The plot also reveals the expected load to the aircraft as a result of transiting the steep gradients of vertical velocity on the flanks of the plume. The 'truth' and radar prediction of load compare well with the load derived from Bowles' model. All sources indicate a severe turbulence event whether from observed data or simulation for the B-757 with configuration similar to the NASA flight on April 30, 2002 (see Table 1).

In this paper, we will examine further the potential of the data sets, the ADWRS simulation system, and the hazard prediction models. Simulation results will be 
presented demonstrating the flexibility of the models to evaluate turbulence affects of a single turbulence field to a variety of aircraft and aircraft configurations; particularly how minimal changes in weight can have on affecting the aircraft load. Furthermore, plans for implementing these tools as an FAA certification methodology "tool set" will be discussed.

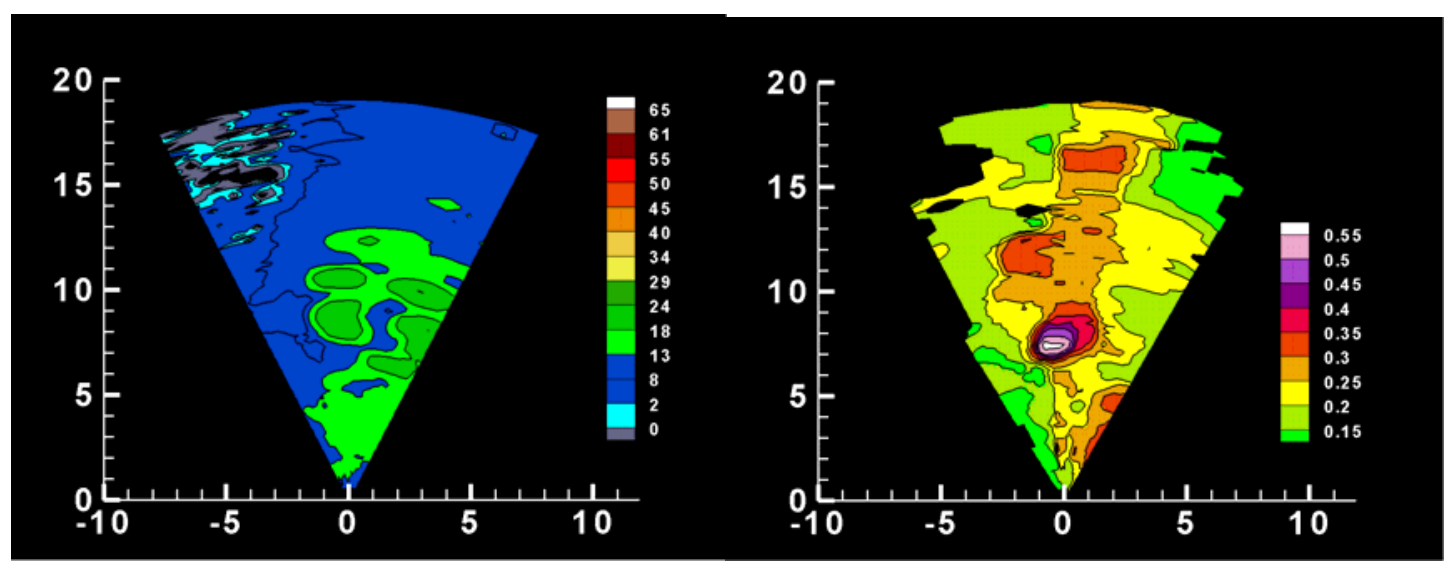

Figure 1. Radar reflectivity ( $\mathrm{dBz}$, left panel) and predicted hazard (right panel) retreived from NASA's B-757 onboard radar at 19:13:20 UTC on 30 April 2002, just prior to encounter (Event 23210). Distance is in kilometers.

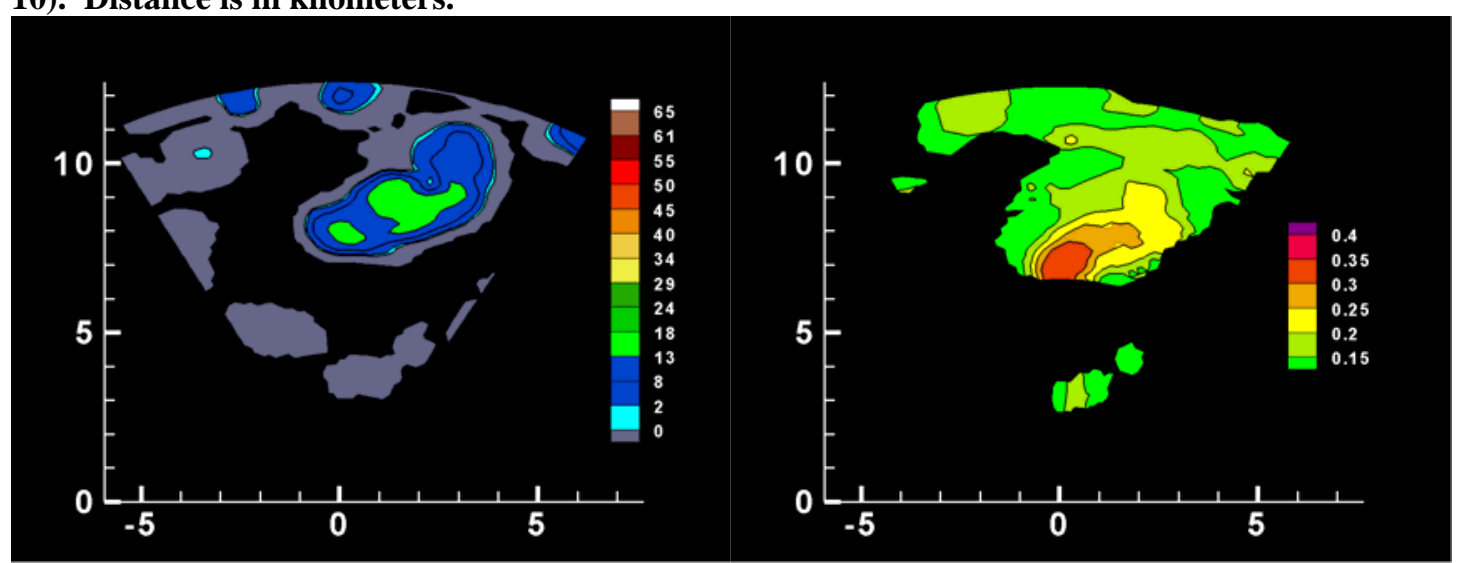

Figure 2. ADWRS simulated radar reflectivity (left panel) and predicted hazard (right panel) from TASS 232-10 data set at an altitude of $10 \mathrm{~km}$ above ground level. The scale is $\sim 1 / 2$ of that shown in Fig.1. 


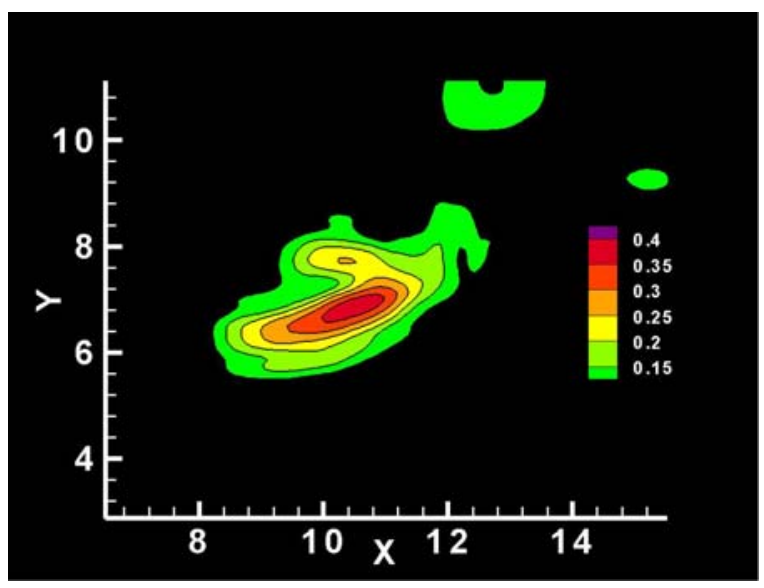

Figure 3. Hazard field derived from the TASS 232-10 data set using the Moving box method.

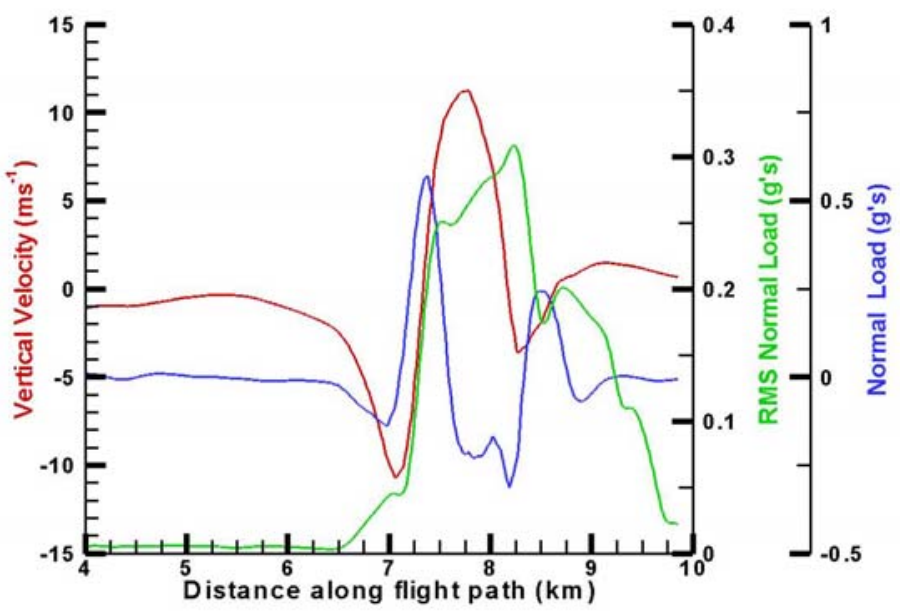

Figure 4. Profiles extracted along a selected flight path in TASS 232-10 data set. Vertical velocity (red), RMS normal load acceleration (green), and normal load acceleration (blue) vs distance along the flight path as computed from the vertical velocity field extracted along an $\mathrm{X}=2$ line in Figure 2. Calculation assumes 5 sec moving average.

Table 1. RMS Normal Load Comparison for Event 232-10.

\begin{tabular}{|lc|}
\hline \multicolumn{1}{|c|}{ Source } & Peak $\sigma_{\Delta \mathrm{n}}(\mathbf{g}$ 's) \\
\hline In situ & 0.44 \\
Onboard Turbulence Radar & 0.55 \\
Flight Dynamics Simulation & 0.31 \\
Moving Box Method & 0.38 \\
Radar Simulation with Model Data & 0.34 \\
\hline
\end{tabular}

\footnotetext{
${ }^{1}$ G. F. Switzer and C. L. Britt, Performance of the NASA Airborne Radar With the Windshear Database for Forward-Looking Systems, NASA CR-201607, September 1996, pp. 85.

${ }^{2}$ Cornman, L.B., Williams, J.K., and Goodrich, R.K., "The Detection of Convective Turbulence Using Airborne Doppler Radars," Preprints, $9^{\text {th }}$ Conf. on Aviation, Range, and Aerospace Meteorology, Orlando, FL, Amer. Meteor. Soc., September 2000, pp. 569-574
} 
${ }^{3}$ Proctor, F.H., Hamilton, D.W., and Bowles, R. L., "Numerical Simulation of a Convective Turbulence Event," Preprints, $10^{\text {th }}$ Conference on Aviation, Range, and Aerospace Meteorology, Portland, OR, Amer. Meteor. Soc., May 2002, pp. 41-44.

${ }^{4}$ Bowles, R.L., "Aircraft Centered Hazard Metric Based on Airborne Radar Turbulence Observables," AeroTech Report ATR-12010, (prepared for NASA Langley Research Center), September 2000.

${ }^{5}$ Proctor, F.H., "The Terminal Area Simulation System, Volume 1: Theoretical Formulation," NASA Contractor Report 4046, DOT/FAA/PM-85/50, 1, April 1987, 176 pp.

${ }^{6}$ Bowles, R.L., personal communication. 


\title{
Airborne Turbulence Detection System Certification Tool Set
}

\author{
David W. Hamilton ${ }^{*}$ and Fred H. Proctor ${ }^{\dagger}$ \\ NASA Langley Research Center, Hampton, Virginia, 23681
}

\begin{abstract}
A certification tool set is developed for supporting the FAA in certification of airborne turbulence detection systems. Candidate scenarios are provided along with a tool set for the performance evaluation of a candidate automated turbulence detection system.
\end{abstract}

$\begin{array}{ll}\text { Nomenclature } \\ \mathrm{L}_{\mathrm{x}} & =\text { distance between } \sigma_{\mathrm{u}} \text { and } \sigma_{\mathrm{w}} \text { along flight path } \\ \mathrm{RRF} & =\text { radar reflectivity factor } \\ \mathrm{u} & =\text { in situ along path wind } \\ \mathrm{W} & =\text { in situ vertical wind } \\ \mathrm{x} & =\text { abscissa; coordinate orthogonal to flight path } \\ \mathrm{y} & =\text { ordinate; coordinate parallel to flight path } \\ \sigma_{\Delta \mathrm{n}} & =\text { Root Mean Square normal load } \\ \sigma_{\mathrm{u}} & =\text { Root Mean Square in situ head wind } \\ \sigma_{\mathrm{w}} & =\text { Root Mean Square in situ vertical wind }\end{array}$

\section{Introduction}

$\mathrm{U}^{\mathrm{n}}$ NEXPECTED encounters with turbulence are the leading cause of in-flight injuries ${ }^{1}$ and have occasionally resulted in passenger and crew fatalities. Most of these injuries are caused by sudden and unexpected encounters with severe turbulence in and around convective activity. ${ }^{2}$ To alleviate this problem and contribute to the mission of NASA's Aviation Safety and Security Program, technologies have been investigated to detect and warn of hazardous in-flight turbulence. Under the sponsorship of the Turbulence Prediction and Warning Systems (TPAWS) element of NASA's Aviation Safety and Security program, a radar-based airborne turbulence detection system (ATDS) has been developed and tested, which improves upon existing commercially available systems. The new system utilizes algorithms and hazard metrics developed for use with existing airborne predictive windshear radar, and warns of hazardous turbulence even in regions with weak radar reflectivity (i.e. 5-15 dBz). NASA's experimental system was flown on a B757 research aircraft and successfully detected hazardous convectively induced turbulence (CIT) with few false alarms. ${ }^{3,4}$ Currently, a similar system is being flown on a commercial airliner. Data obtained from that system show promising results as well.

Industry is expected to market similarly improved ATDS components in the near future. Since the new systems may incorporate alerting functionality, requiring flight crews to take procedural action in the event of a potentially hazardous turbulence encounter, industry is expected to file for FAA certification. To prepare for this activity, the FAA has developed requirements for certification. NASA has provided guidance in the development of these requirements and furthermore, developed a certification methodology, an accompanying tool set, and test criteria to evaluate the performance of industry built ATDS. In this paper, we will present the certification tool set and example simulation test criteria that has been made available to the FAA for assisting in the certification of future radar-based ATDS.

\section{Certification Requirements and Methodology}

The purpose of a certification process is to establish a performance standard for future ATDS. Performance standards have been established using a metric that describes the aircraft's response to turbulence, i.e. the aircraft normal load. Turbulence measurements are quantified in terms of a 5 second moving average of the normal load, or the RMS normal loads $\left(\sigma_{\Delta \mathrm{n}}\right)$, where $\sigma_{\Delta \mathrm{n}}<0.20 \mathrm{~g}$ is considered light, $0.20 \mathrm{~g} \leq \sigma_{\Delta \mathrm{n}}<0.30 \mathrm{~g}$ is considered moderate,

\footnotetext{
* Aerospace Technologist, Aviation Operations and Evaluation Branch, Mail Stop 156A.

${ }^{\dagger}$ Aerospace Technologist, Aviation Operations and Evaluation Branch, Mail Stop 156A, and AIAA Senior Member. 


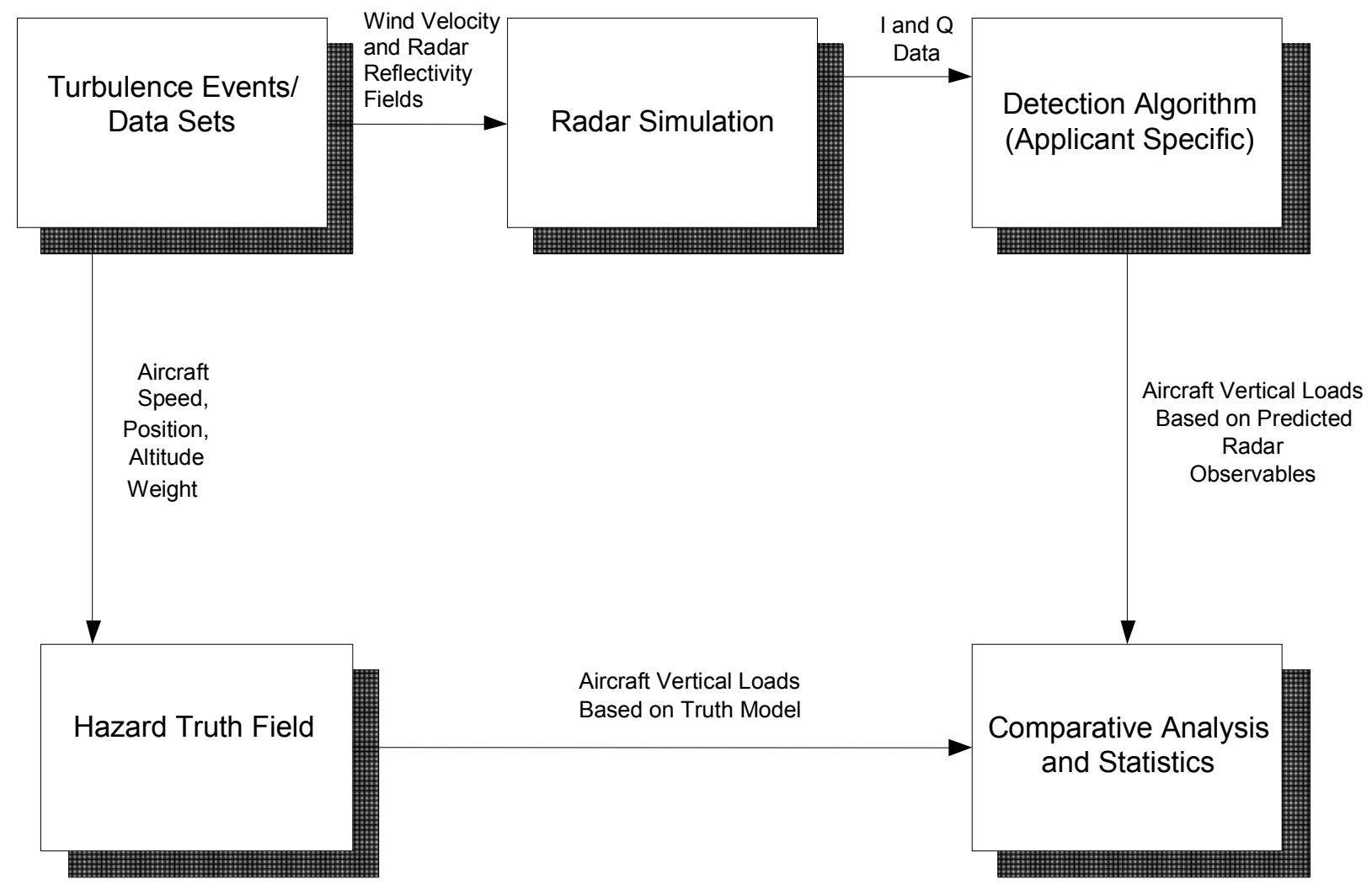

Figure 1. Schematic representation of the certification methodology that details all components necessary for certification of an ATDS alerting system

and $\sigma_{\Delta \mathrm{n}} \geq 0.30 \mathrm{~g}$ is severe. Since the primary operational goal is to provide the flight crew with a situation display of severe convective turbulence ahead of the aircraft, these general requirements have been set forth by the FAA: 1) the ATDS will detect and provide for the display of severe convective turbulence, with reflectivity greater than 15 $d B z$ at a minimum of 5 nmiles $85 \%$ of the time and 2) the ATDS will not indicate severe convective turbulence that would produce a $\sigma_{\Delta \mathrm{n}}$ value less than $0.10 \mathrm{~g}$ greater than $20 \%$ of the time. Hereafter, the general requirements just described will be referred to as "must display" and "must not display", respectively.

A combination of analysis and simulation will be required to demonstrate compliance with these general requirements. A methodology has been established as the basis for the simulation efforts in the certification process. The certification methodology is described in Figure 1.

The methodology in Figure 1 identifies the components of an end-to-end simulation system used to evaluate the performance of a candidate ATDS. Each component in Figure 1 has been addressed by separate but parallel TPAWS research activities. The resulting simulation system, or 'tool set' can be used to evaluate the performance of a candidate radar-based hazard detection algorithm that is being considered for FAA certification. The tool set simulates an airborne predictive windshear (PWS) radar that is directed into a predetermined turbulence environment. In a hypothetical evaluation, a user applies the tool set by conducting a flight path through a threedimensional gridded atmospheric data set representing convectively induced turbulence (CIT). The user specifies the initial aircraft parameters (type, speed, altitude, and weight) and the radar simulation creates the inphase and quadrature (I and Q) signal from the wind velocity and radar reflectivity data within the CIT data set. The I and Q data is then processed by the user's hazard detection algorithm to produce a $\sigma_{\Delta \mathrm{n}}$ prediction. The prediction is validated by known characteristics of the gridded CIT data set requiring a "truth" field. Scoring the detected hazard utilizes criteria derived from flight data for assuring acceptable performance of the candidate hazard detection algorithm. 


\section{Certification "Tool Set"}

Each component of the tool set is described here in detail. The tool set consists of model-generated atmospheric turbulence data sets ${ }^{6,7,8}$, a hazard estimation model to produce a "truth" field, a radar simulation software, and aircraft hazard tables required for converting the radar observable to a hazard prediction. These tools provide the controlled environment necessary for such an evaluation. In a following section, the tools will be used to demonstrate flight path scenarios made available to the FAA for addressing the "must display" and "must not display" requirements.

\section{A. Turbulence Events/Data Sets}

The effort in developing the tool set has required the selection of events where aircraft have encountered hazardous CIT. Events were chosen for turbulence encounters involving both commercial and research aircraft. ${ }^{3-8}$ The turbulence environments associated with each event have been simulated with an atmospheric numerical model. The numerical model generates data sets of the turbulence environments, which have been validated with observations.

The data sets were generated with NASA's Terminal Area Simulation System ${ }^{9,10}$ (TASS). TASS is an atmospheric large eddy simulation initialized with the preconvective environment observed in the vicinity of the actual event. The resulting data sets, along with appropriate user guides, have been made available for use by radar simulation software and the subsequent testing of candidate ATDS load prediction algorithms.

Data sets include cases with hazardous levels of CIT, as well as benign cases. The most recent data set has relatively benign turbulence characteristics, supporting the "must not display" scenario in the certification process. Although details of the data set will not be discussed in this paper, the "must not display" scenario will be demonstrated.

The four data sets include simulated environments for; 1) NASA's B-757 turbulence event 191-06, which occurred on December 14, 2000, 2) NASA's turbulence event 232-10, which occurred on April 30, 2002, 3) an event involving a Flight Operations and Quality Assurance flight of a B-737 over Delaware, and 4) a high plains thunderstorm observed during a meteorological field experiment. ${ }^{11,12}$

\section{B. Radar Simulation}

A radar simulation system can ingest the CIT data sets, and generate I and Q inputs for the hazard detection algorithm. The radar simulation tool used in the proposed methodology was initially developed for NASA's Airborne Windshear Program and is called the Airborne Doppler Weather Radar Simulation (ADWRS). ${ }^{13,14}$ With ADWRS, flight paths can be defined for a variety of aircraft and aircraft conditions to generate I and Q for the required hazard predictions.

\section{Radar Algorithm}

The radar hazard detection algorithm is the component that computes the turbulence hazard. Industry is responsible for developing the hazard detection algorithm and demonstrating the feasibility of their system. For demonstration purposes, the algorithm demonstrated in this paper is the same that was used on the NASA flight tests.

\section{Aircraft "Truth" Fields}

A "truth" field is required to compare the turbulence hazard predicted by the detection algorithm with the actual hazard in the gridded data set. The aircraft turbulence truth field is obtained from the modeled data sets by computing a moving box standard deviation of the vertical wind field. ${ }^{6,8}$ The calculation uses the vertical wind field in the horizontal plane consistent with the aircraft altitude. The spatial bounds of the moving box are computed as the distance the aircraft moves through the wind field in 5 seconds. The bounds are applied as a 2.5 second distance ahead, behind, and to the sides of the current position. Once the standard deviation of the vertical wind is computed, a scale factor is applied to compute $\sigma_{\Delta \mathrm{n}}$. Bowles has developed an array of hazard tables that provide this scale factor for converting the radar detected hazard to $\sigma_{\Delta \mathrm{n}}{ }^{15,16}$ The hazard tables consider aircraft specific criteria including type, weight, speed, and altitude.

\section{E. Comparative Analysis and Statistics}

Finally, a comparison between the detected and 'truth' hazard is necessary to evaluate the candidate detection algorithm performance. This may not be straight forward since the detected hazard may either be displaced from the actual hazard, differ in magnitude, or both, thus requiring certain criteria for scoring a detection. For this, NASA 


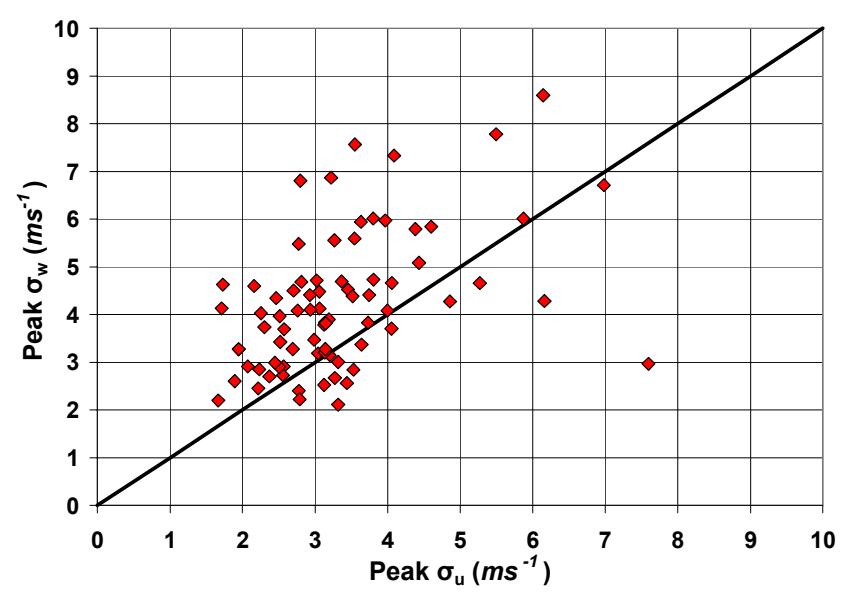

Figure 2. Corresponding peak values of headwind variance $\left(\sigma_{u}\right)$ and vertical wind variance $\left(\sigma_{w}\right)$ measured in situ for NASA's turbulence events. Variances were computed using a 5 second window.

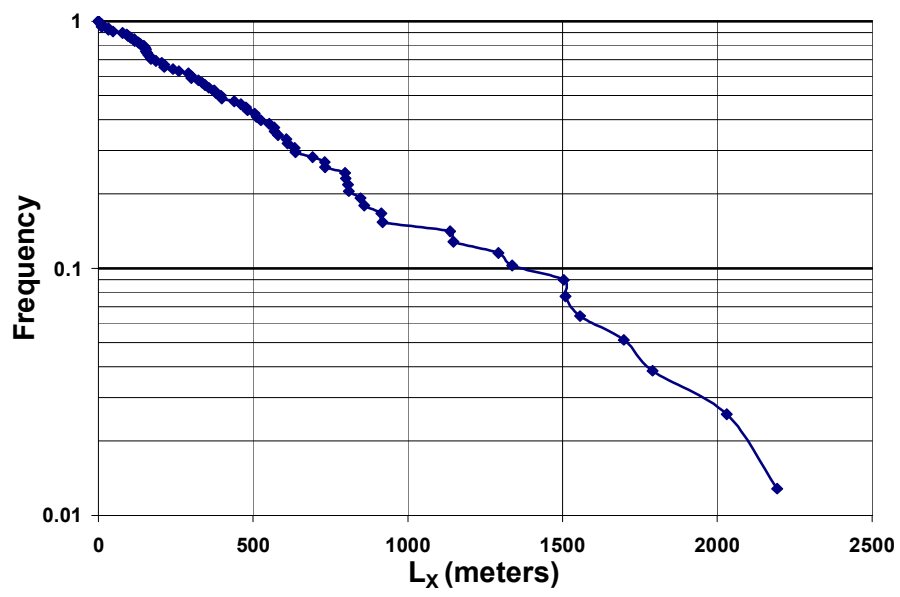

Figure 3. Frequency distribution of the distance between peak values of $\sigma_{u}$ and $\sigma_{\mathrm{w}}$ for events presented in Figure 2.

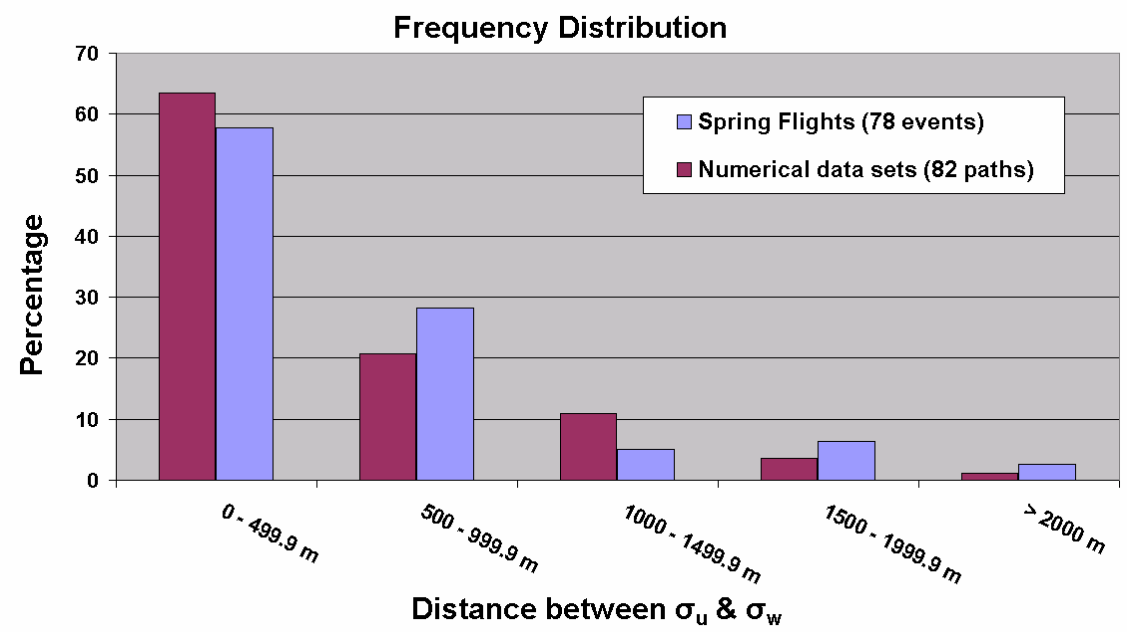

Figure 4. Comparison of distances between $\sigma_{u}$ and $\sigma_{w}$ from flight data and paths through data sets. 
flight test data is used to examine characteristics of CIT and apply the results to developing scoring criteria for ATDS., ${ }^{3,4,5,8}$

A weakness of turbulence detection with radar is that the radar senses air motion along the radial coordinates of the radar cone; whereas the aircraft primarily responds to vertical air motion along the flight path. Therefore, assumptions must be made in order to convert radar measurements into an aircraft hazard metric. If atmospheric turbulence were truly isotropic at the scale of the radar pulse volume, then one would expect perfect correlation between the statistics of these two wind components. However, in situ data collected by NASA's B-757 in 2000 and 2002 indicates that CIT is often anisotropic. To demonstrate the radar challenge, peak variances were calculated for in situ measurements of vertical wind and along path wind components. The data shows peak values of $\sigma_{u}$ and $\sigma_{\mathrm{w}}$ rarely had the same value (Figure 2) and were not always located at the same point within the same turbulence patch (Figure 3). In most of the events, values for $\sigma_{\mathrm{w}}$ are greater than $\sigma_{\mathrm{u}}$. For radar detection, this may imply the possibility of an underprediction of the magnitude of turbulence intensity. Therefore, one needs to consider magnitude buffers for display thresholds.

The NASA flight test data supports the need for a spatial buffer, as well. For most of the events, the spatial displacement between peak $\sigma_{\mathrm{u}}$ and $\sigma_{\mathrm{w}}$ is greater than $400 \mathrm{~m}$ (Figure 3). This spatial displacement between detected $\left(\sigma_{\mathrm{u}}\right)$ and true $\left(\sigma_{\mathrm{w})}\right.$ hazard should not be a problem if buffers are placed around the true hazard. Note that in nearly all of the events the displacement was less than $2 \mathrm{~km}$. Similarly, data extracted from flight paths through the gridded data sets show comparable displacements (see Figure 4). Therefore, an alerting strategy that considers up to a $2 \mathrm{~km}$ displacement (spatial buffer) between the boundaries of the sensed and actual hazard seems to be appropriate.

\section{Tool Set Application}

Two simulations are presented here to demonstrate the tool set applicability in the certification of ATDS systems. Turbulence hazard predictions are made using the hazard algorithm that was used on the NASA flight experiments, the NCAR Efficient Spectral Processing Algorithm (NESPA) ${ }^{17}$.

In the first demonstration, a "must display" scenario will be presented, i.e. a case where severe turbulence is present ahead of the aircraft. The event chosen is a turbulence encounter that occurred during one of NASA's flight experiments; Event 232-10. Aircraft parameters for the simulation represent a B-757 with a weight of 200,000 lbs, an altitude of 33,000 feet, and an airspeed of $200 \mathrm{~ms}^{-1}$. Simulated radar reflectivity and predicted hazard are shown in Figure 5 and Figure 6, respectively. The peak hazard magnitude and location compare well with the 'truth' depicted in Figure 7.

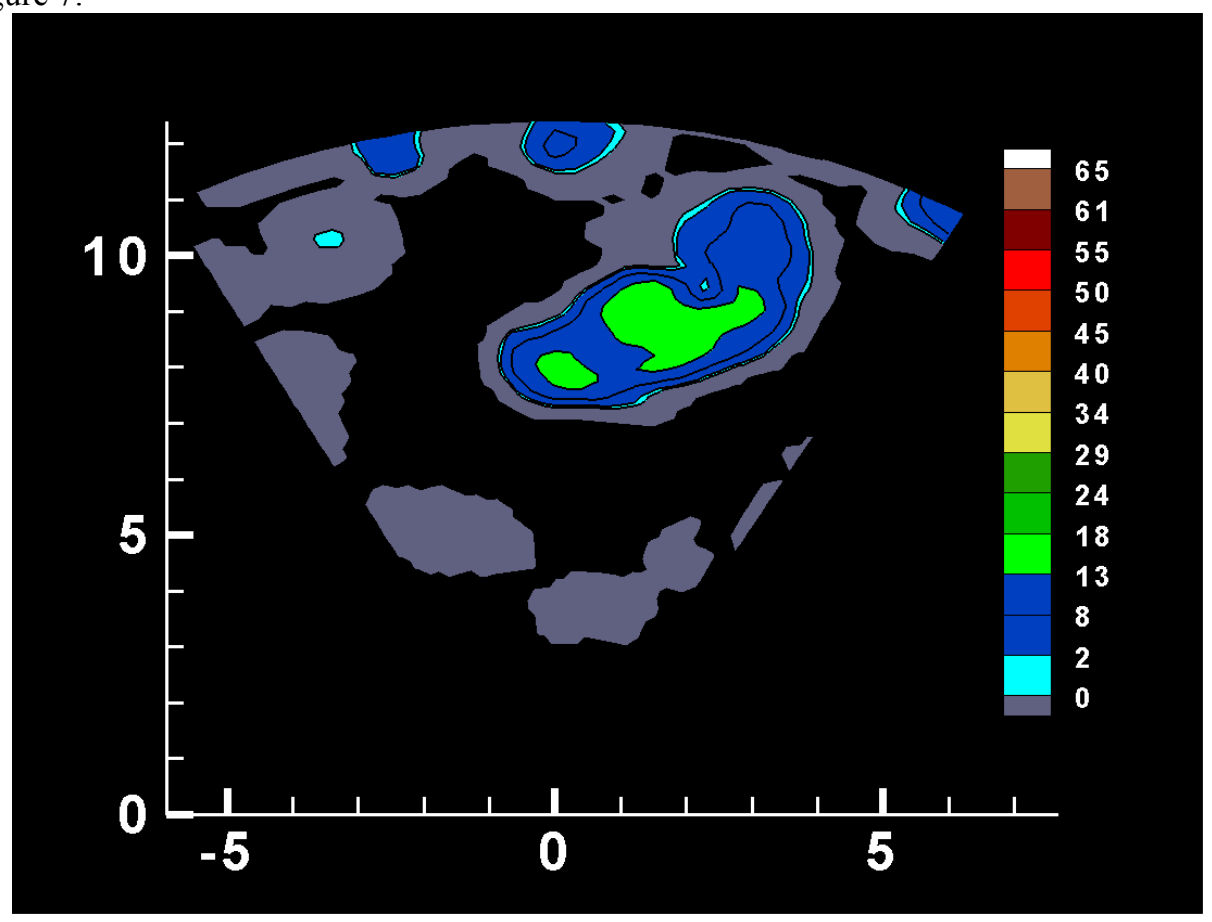

Figure 5. ADWRS simulated radar reflectivity in $d B z$. Distance is in kilometers relative to the aircraft position. 


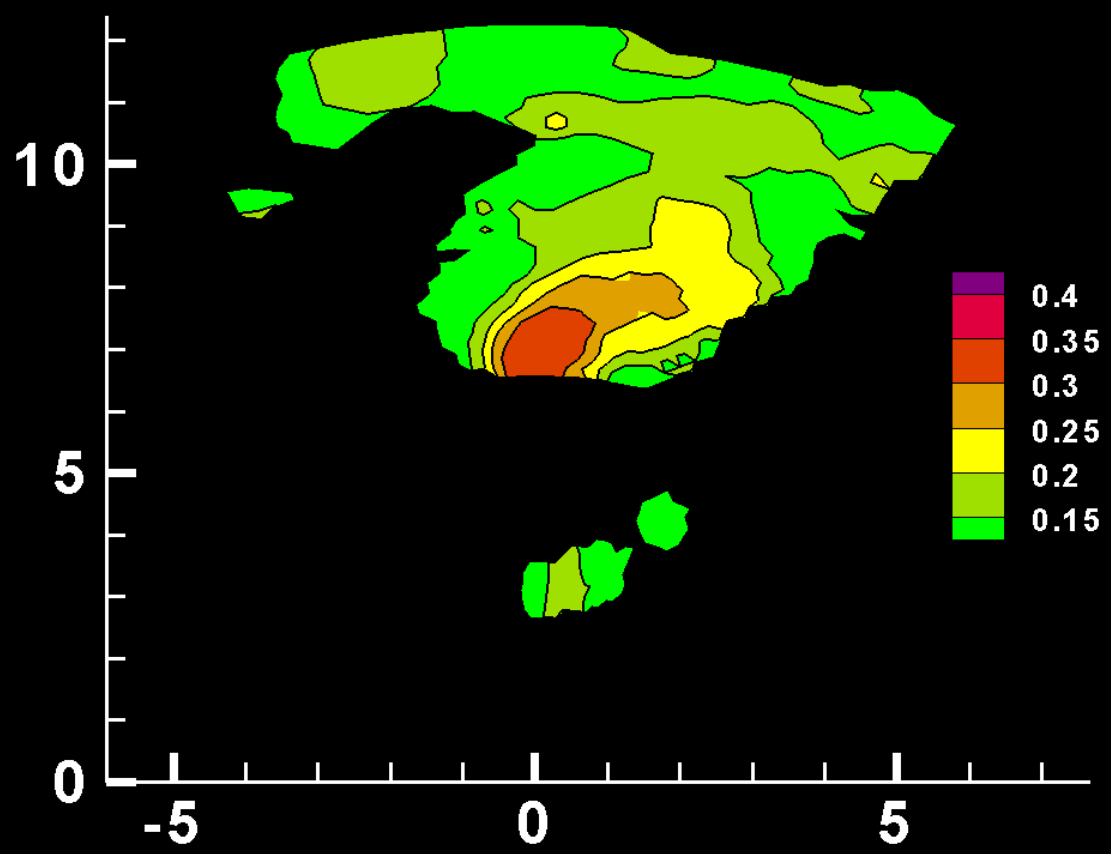

Figure 6. Same as Figure 5, but for predicted $\sigma_{\Delta \mathrm{n}}\left(\mathrm{g}^{\prime} \mathrm{s}\right)$.

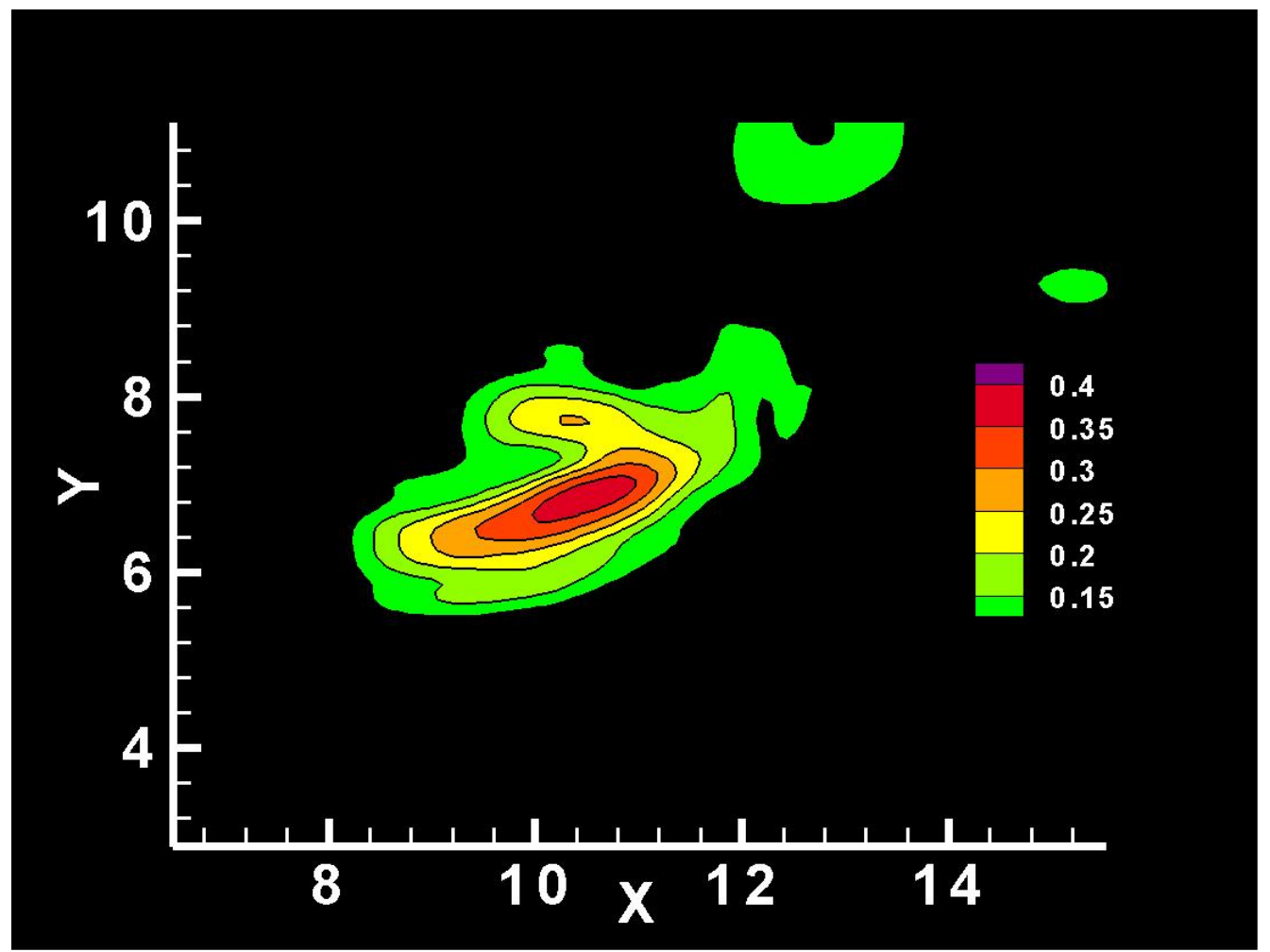

Figure 7. Truth field derived from the TASS 232-10 data set using the Moving box method. Distance is in kilometers relative to the data set origin. 
For the scoring exercise, radar reflectivity and turbulence hazard are depicted similar to that of an airborne radar display (Figure 8-11). A typical airborne radar display for commercial aircraft shows a three level radar reflectivity (20,30 and $40 \mathrm{dBz}$ contours) and a single level turbulence hazard (flooded or speckled magenta). In the figures an additional contour for $15 \mathrm{dBz}$ is added due to the low reflectivity nature associated with the turbulence region. The turbulence hazard is depicted for moderate and severe turbulence. The purpose here is to demonstrate the scoring criterion described in Section III.E.

Truth field and radar simulation results are presented in Figure 8 and Figure 9, respectively. The spatial buffer of $2 \mathrm{~km}$ has been approximated in Figure 10 and Figure 11 showing the overlap with the truth and detected hazard fields. The detection algorithm indicates moderate turbulence whereas the truth field indicates severe turbulence. Although the peak hazard magnitudes differ by nearly $0.1 \mathrm{~g}$, the detected turbulence should be displayed. This suggests the need for a magnitude buffer.

In the second demonstration, a "must not display" scenario is presented, i.e. a case where severe turbulence must not be displayed when $\sigma_{\Delta \mathrm{n}}$ remains less than $0.1 \mathrm{~g}$ near the flight path. The event chosen for this case is a data set simulating a high plains thunderstorm, referred to as the Miles City data set. Aircraft parameters for the simulation represent a B-757 with a weight of 200,000 lbs, an altitude of 22,000 ft above ground level, and an airspeed of 215 $m s^{-1}$.

Truth field and radar simulation results are shown in Figure 12 and Figure 13, respectively. The truth field has a peak $\sigma_{\Delta \mathrm{n}}$ value of $0.06 \mathrm{~g}$, which is below the display threshold for moderate turbulence (Figure 12). This truth hazard value is also sufficiently less than the required $0.1 \mathrm{~g}$ threshold for "must not display". Since the hazard algorithm does not indicate severe turbulence along the flight path (Figure 13), the detection is scored a correct null.

\section{Summary}

A methodology and a corresponding set of simulation tools for testing and evaluating turbulence detection sensors has been presented. The tool set is available to industry and the FAA for certification of radar based airborne turbulence detection systems. The tool set consists of simulated data sets representing convectively induced turbulence, an airborne radar simulation system, hazard tables to convert the radar observable to an aircraft load, documentation, a hazard metric "truth" algorithm, and criteria for scoring the predictions. Analysis indicates that flight test data supports spatial buffers for scoring detections. Also, flight data and demonstrations with the tool set suggest the need for a magnitude buffer.

\section{Acknowledgments}

This research was sponsored by NASA's Aviation Safety and Security Program. 


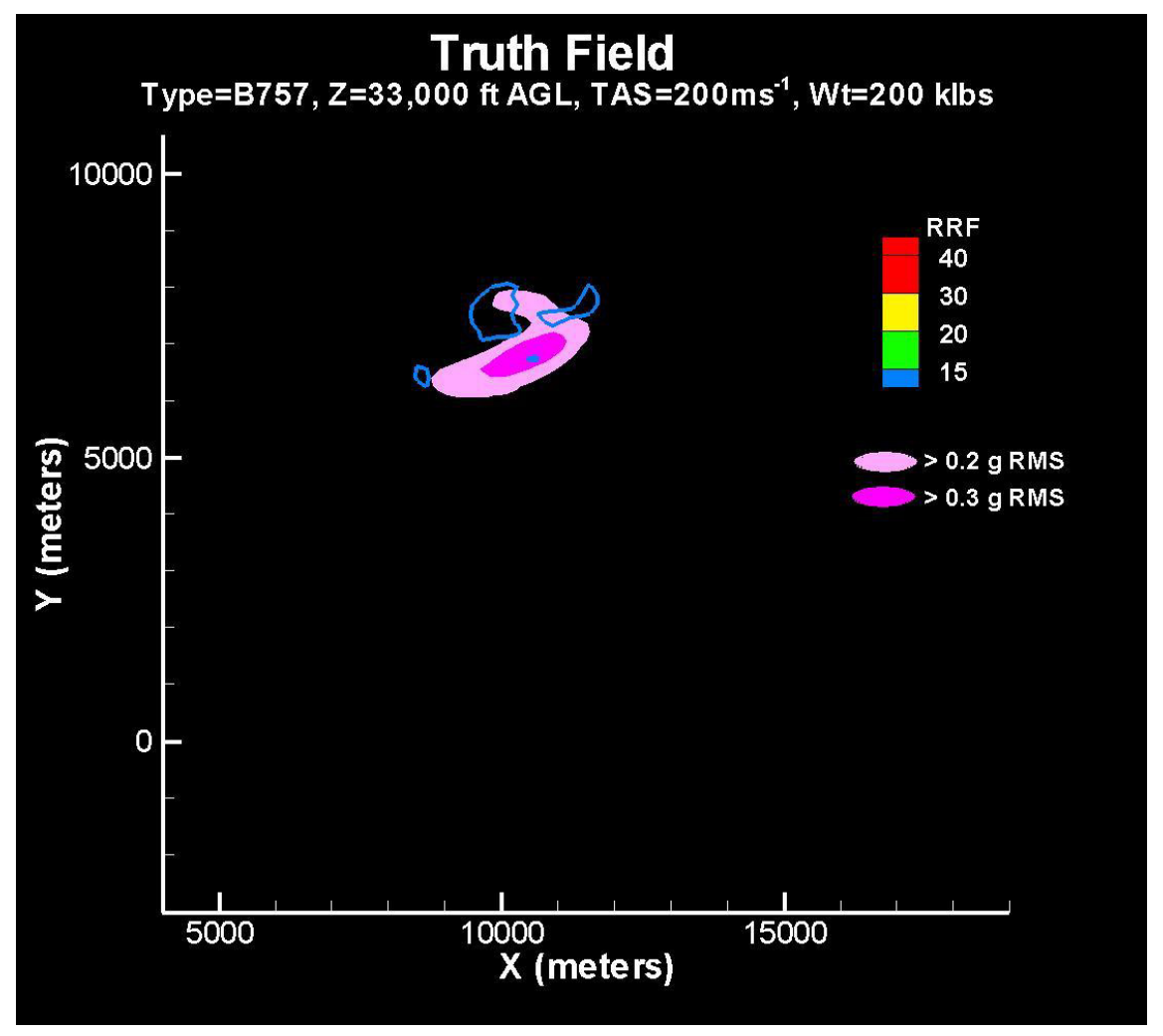

Figure 8. Radar reflectivity (line contours) and truth field (flooded contours) for 232-10 data set. Hazard is contoured for moderate (light magenta) and severe (dark magenta) turbulence intensities.

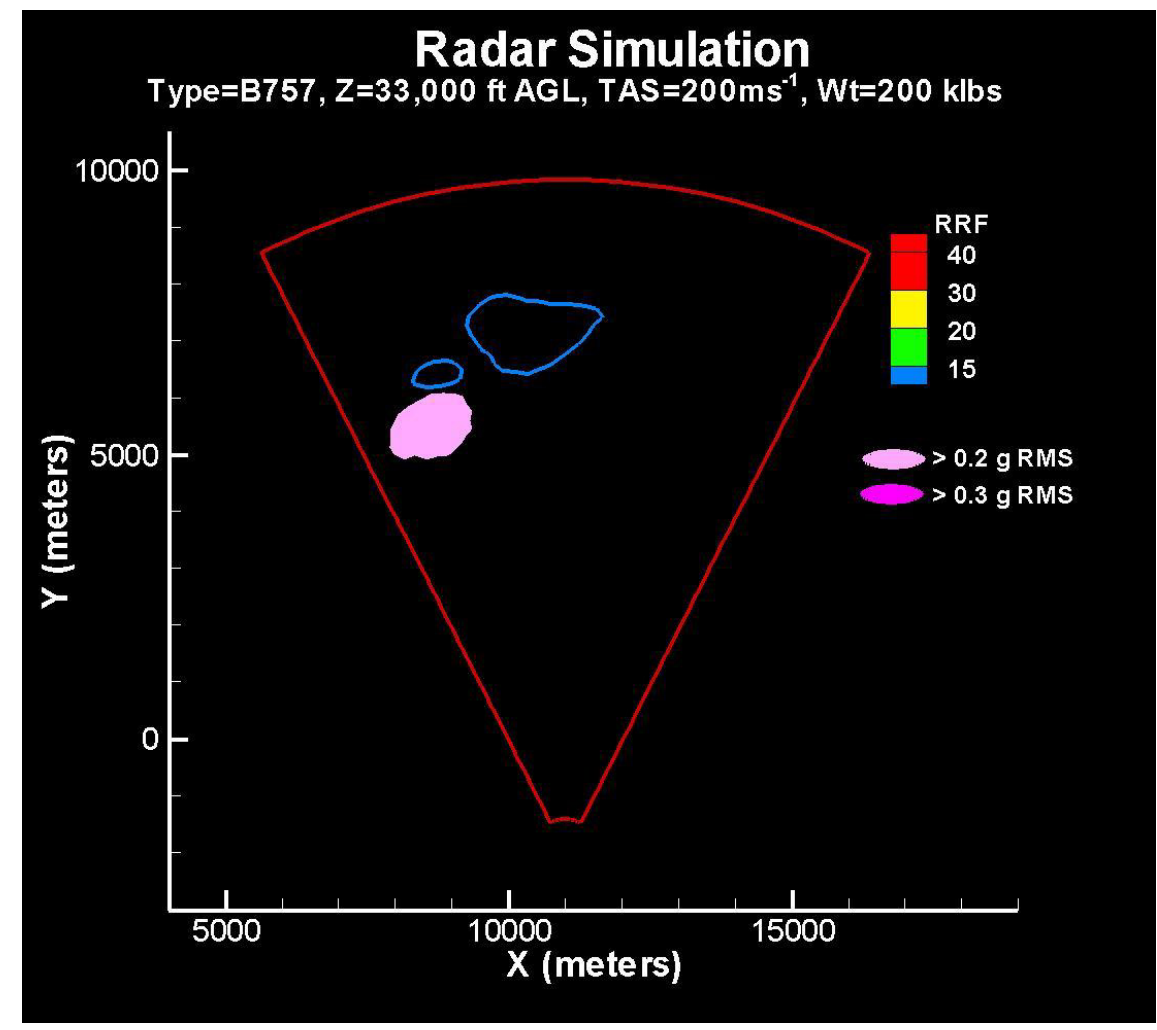

Figure 9. Same as in Figure 8 but for ADWRS/NESPA simulation of Event 232-10. 


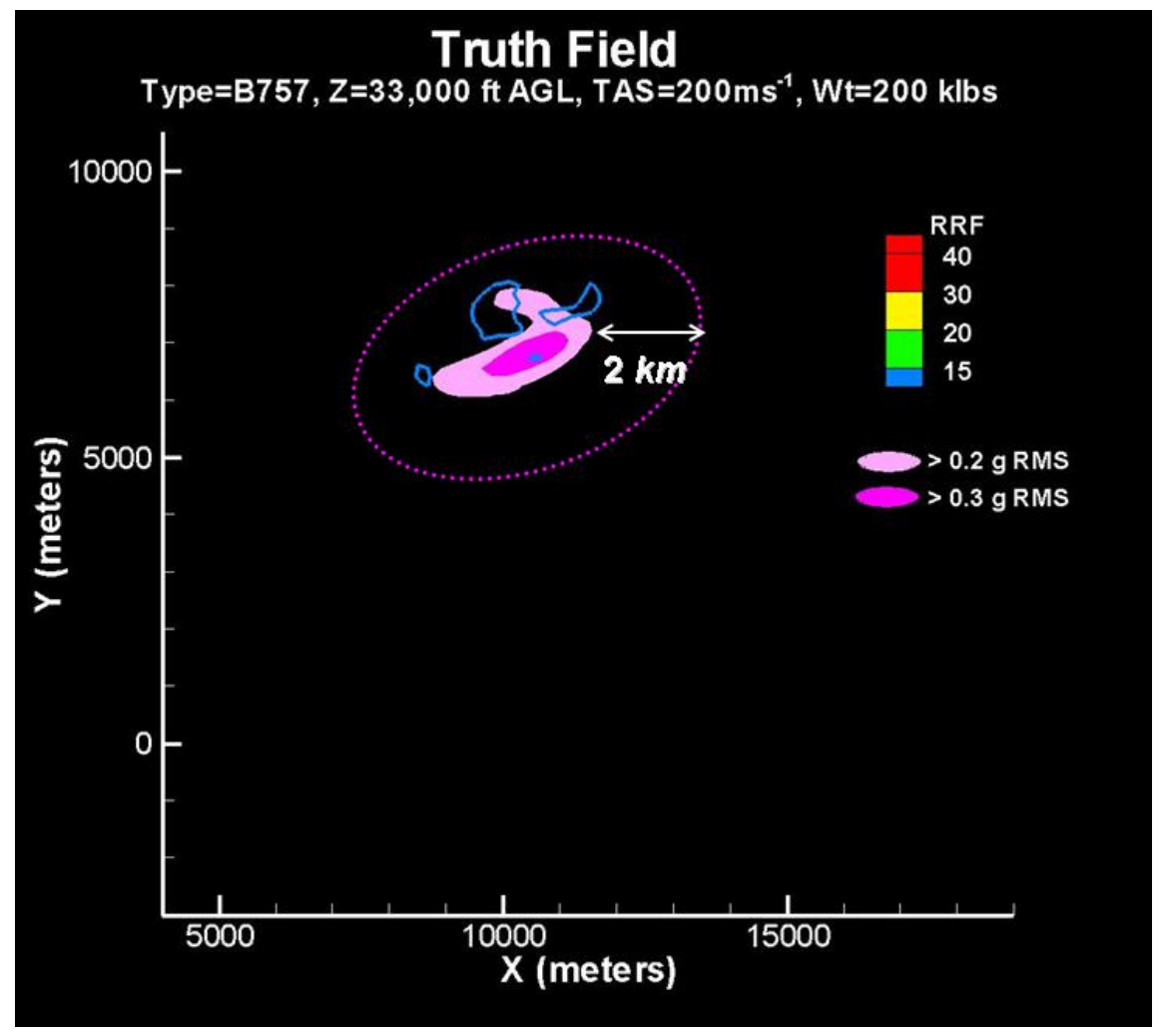

Figure 10. Same as in Figure 8, but with a $2 \mathrm{~km}$ spatial buffer around moderate turbulence boundary.

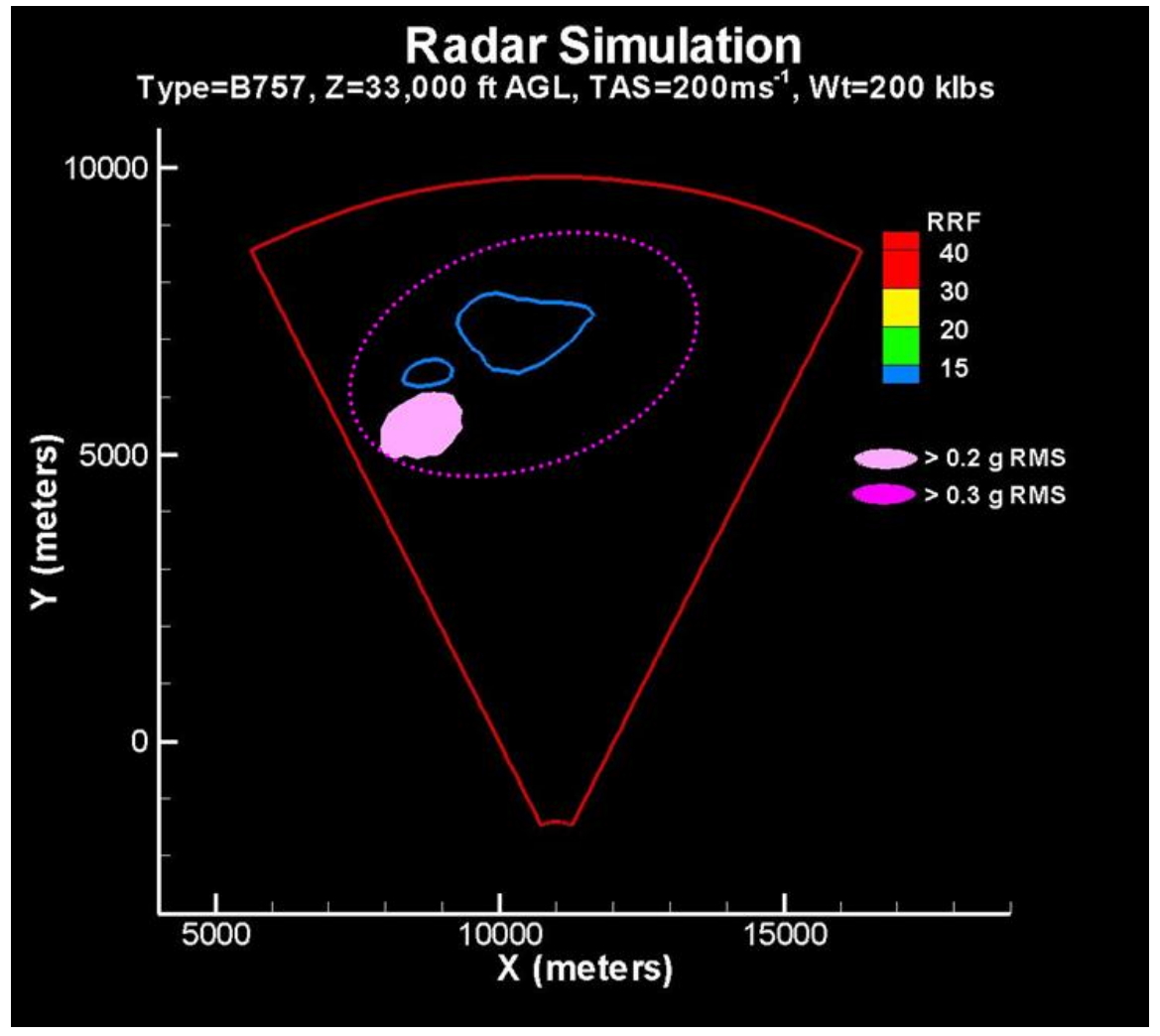

Figure 11. Same as in Figure 9, but with a $2 \mathrm{~km}$ spatial buffer around moderate turbulence boundary. 


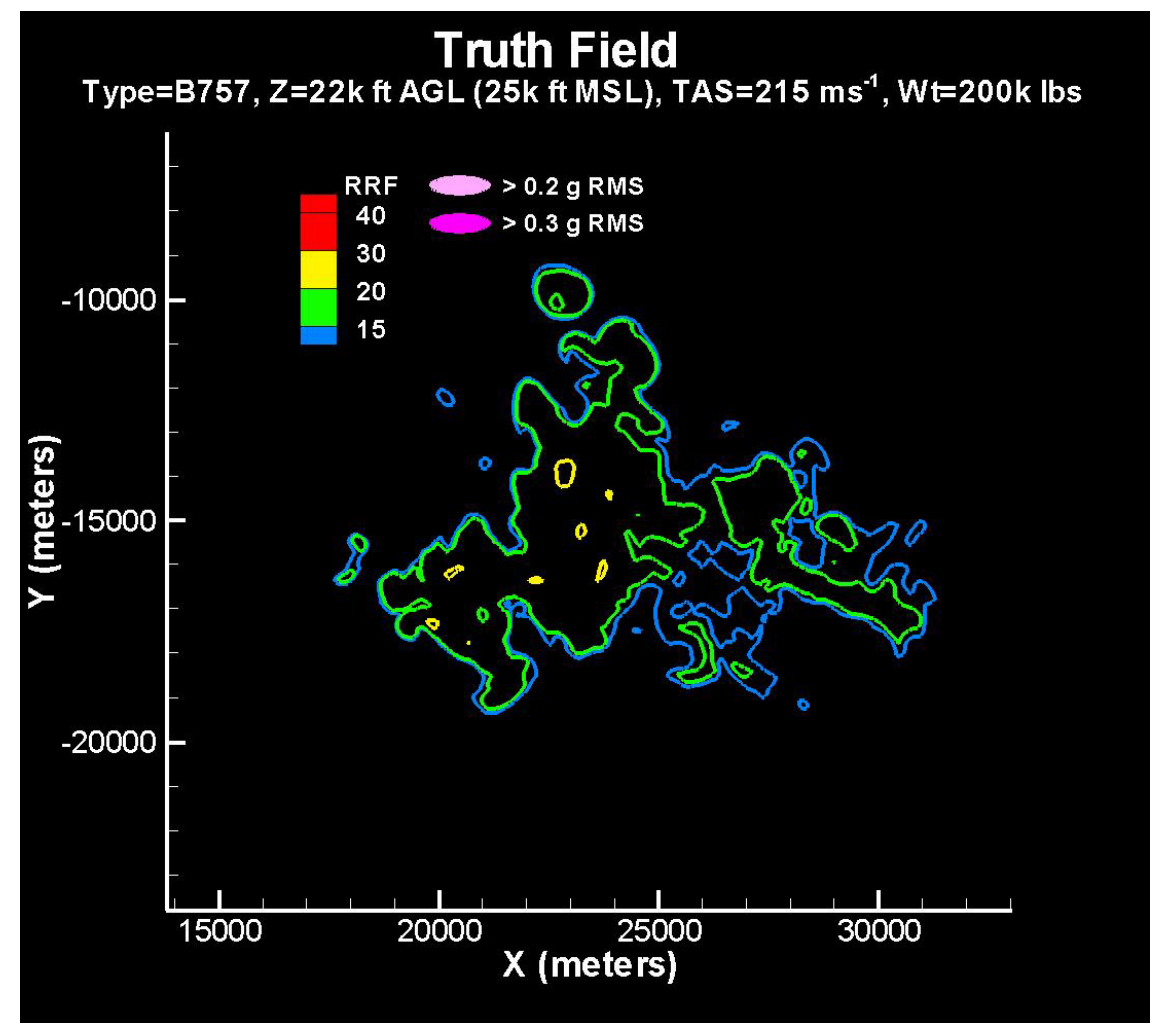

Figure 12. Same as in Figure 8 but for a simulation using the Miles City data set.

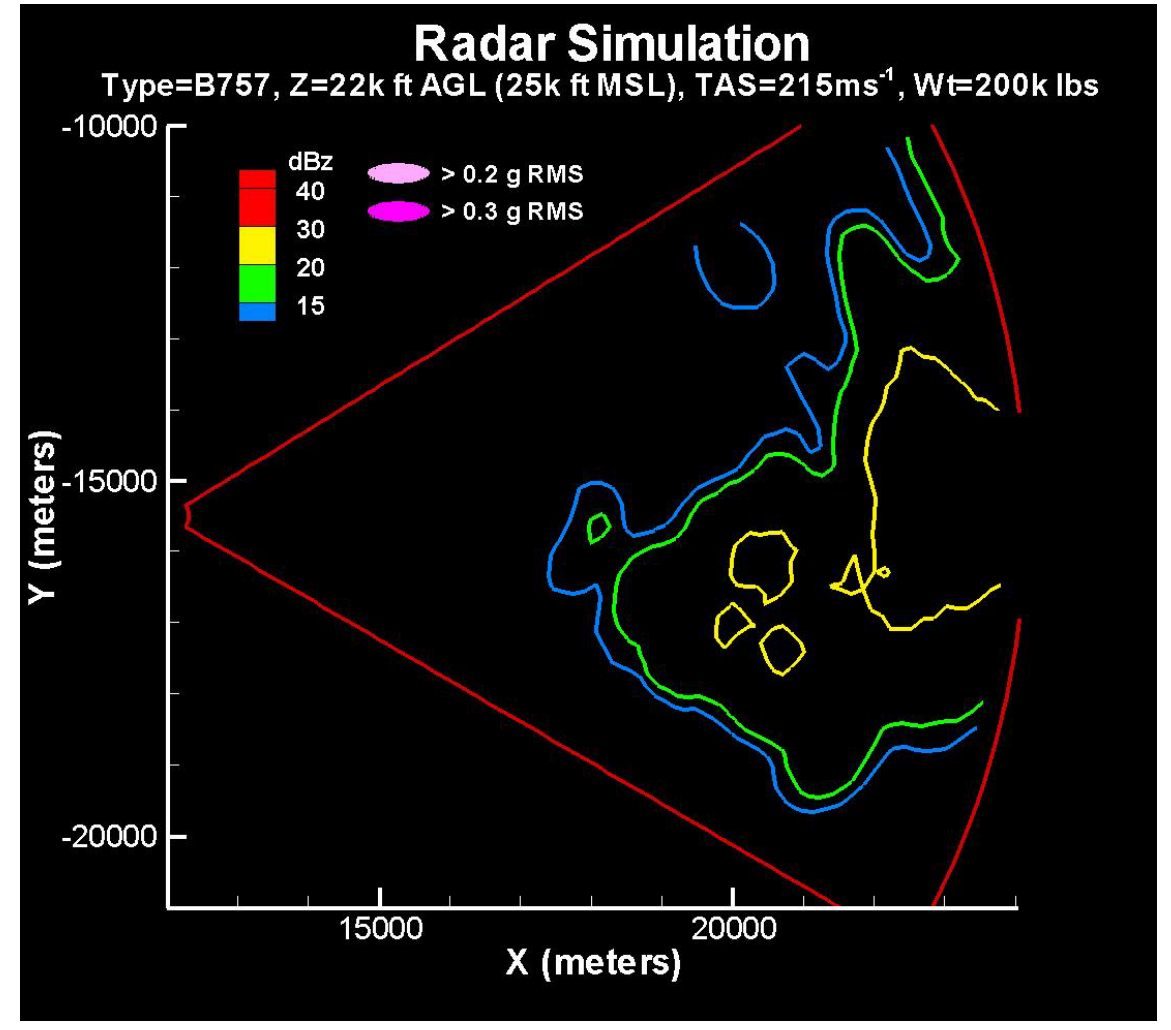

Figure 13. Same as in Figure 9 but for a simulation using Miles City data set. 


\section{References}

${ }^{1}$ Tvaryanas A.P., "Epidemiology of Turbulence-Related Injuries in Airline Cabin Crew, 1992-2001," Aviat Space Environ Med, Vol. 74, 2003, pp. 970-976.

${ }^{2}$ Kaplan, M.L., Huffman, A.W., Lux, K.M., Charney, J.J., Riordan, A.J., and Lin, Y.-L., "Characterizing the Severe Turbulence Environments Associated with Commercial Aviation Accidents," Meteorol.Atmos.Phys., Vol. 88, 2005, pp. 129-152.

${ }^{3}$ Hamilton, D.W., and Proctor, F.H., "Progress in the Development of an Airborne Turbulence Detection System," $12^{\text {th }}$

Conference on Aviation, Range, and Aerospace Meteorology, Amer. Meteor. Soc., Atlanta, Georgia, Jan. 2006,9 pp.

${ }^{4}$ Hamilton, D.W., and Proctor, F.H., "Meteorology Associated with Turbulence Encounters During NASA's Fall-2000 Flight

Experiments," $40^{\text {th }}$ Aerospace Sciences Meeting and Exhibit, AIAA-2002-0943, Reno, NV, January 2002, 11pp.

${ }^{5}$ Hamilton, D.W., and Proctor, F.H., "Convectively Induced Turbulence Encounters During NASA's Fall 2000 Flight

Experiments," $10^{\text {th }}$ Conference on Aviation, Range, and Aerospace Meteorology, Amer. Meteor. Soc., Portland, OR, May 2002 , pp. 371-374.

${ }^{6}$ Proctor, F.H., Hamilton, D.W., and Bowles, R.L., "Numerical Study of a Convective Turbulence Encounter," $40^{\text {th }}$ Aerospace Sciences Meeting and Exhibit, AIAA-2002-0944, Reno, NV, January 2002, 14pp.

${ }^{7}$ Proctor, F.H., Hamilton, D.W., and Bowles, R. L., "Numerical Simulation of a Convective Turbulence Event," $10^{\text {th }}$

Conference on Aviation, Range, and Aerospace Meteorology, Amer. Meteor. Soc., Portland, OR, May 2002, pp. 41-44.

${ }^{8}$ Hamilton, D.W., and Proctor, F.H.,. An Aircraft Encounter with Turbulence in the Vicinity of a Thunderstorm. $21^{s t} A I A A$ Applied Aerodynamics Conference, AIAA-2003-4075, Orlando, FL, June 2003, 11pp.

9Proctor, F.H., "The Terminal Area Simulation System, Volume 1: Theoretical Formulation," NASA Contractor Report 4046, DOT/FAA/PM-85/50, 1, April 1987, 176 pp.

${ }^{10}$ Proctor, F.H., "Numerical Simulation of Wake Vortices During the Idaho Falls and Memphis Field Programs," $14^{\text {th }}$ AIAA

Applied Aerodynamics Conference, Proceedings, Part-II, New Orleans, LA, AIAA-96-2496, June 1996, pp. $943-960$.

${ }^{11}$ Knight, C. A. (Ed.), "The Cooperative Convective Precipitation Experiment (CCOPE), 18 May - 7 August 1981," Bull. Amer. Meteor. Soc., Vol. 63, 1982, pp. 386-398.

${ }^{12}$ Dye, J. E., Jones, J. J., Winn, W. P., Cerni, T. A., Gardiner, B., Lamb, D., Pitter, R. L., Hallett, J., and Saunders, C. P. R., "Early electrification and precipitation development in a small isolated Montana cumulonimbus," J. Geophys. Res., Vol. 91, 1986, pp. 1231-1246.

${ }^{13}$ Arbuckle, P. D., Lewis, M. S., and Hinton, D. A., "Airborne Systems Technology Application to the Windshear Threat ," 20th Congress of the International Council of the Aeronautical Sciences, Sorrento, Italy, ICAS Paper No. 96-5.7.1, September 813, 1996, pp. 1640-1650.

${ }^{14}$ Switzer G. F., and Britt, C. L., "Performance of the NASA Airborne Radar With the Windshear Database for ForwardLooking Systems," NASA CR-201607, September 1996, pp. 85.

${ }^{15}$ Bowles, R.L., 2003: Hazard Metric Data. Available at:

http://tpaws.larc.nasa.gov/flight_data/TPAWS_Certification_Tool_Set/Hazard_Metric_Data/

${ }^{16}$ Bowles, R.L., "Aircraft Centered Hazard Metric Based on Airborne Radar Turbulence Observables,” AeroTech Report ATR-12010, (prepared for NASA Langley Research Center), September 2000.

${ }^{17}$ Cornman, L.B., Williams, J.K., and Goodrich, R.K., "The Detection of Convective Turbulence Using Airborne Doppler Radars," $9^{\text {th }}$ Conf. on Aviation, Range, and Aerospace Meteorology, Orlando, FL, Amer. Meteor. Soc., September 2000, pp. 569574. 